\title{
Crystallographic and biophysical studies of interactions of new $\beta$-lactoglobulin variants with tricyclic drugs
}

\author{
Paulina Wróbel ${ }^{1}$, Joanna Loch ${ }^{1}$, Piotr Bonarek ${ }^{2}$, Krzysztof Lewiński $^{1}$ \\ ${ }^{1}$ Jagiellonian University, Faculty of Chemistry, Department of Crystal Chemistry and Crystal Physics, \\ Gronostajowa 2, 30-387 Krakow, Poland \\ ${ }^{2}$ Jagiellonian University, Faculty of Biochemistry, Biophysics and Biotechnology, Department of Physical Biochemistry, \\ Gronostajowa 7, 30-387 Krakow, Poland \\ paulina.wrobel@doctoral.uj.edu.pl
}

$\beta$-Lactoglobulin (BLG) is a protein from the lipocalin family [1]. BLG is a milk protein with a natural affinity to fatty acids and retinol [2], however, it can bind with relatively high affinity but rather low selectivity a variety of biomolecules [3]. A conserved structural element of lipocalins is the eight-stranded antiparallel $\beta$-barrel which is the primary binding site for ligands [4]. Like many proteins from the lipocalin family, BLG can be modified by rational site-directed mutagenesis. Substitutions in the region of the $\beta$-barrel can be used to re-design the shape of the binding pocket to create new BLG variants with specific ligand preferences [5]. New variants (mutants) of $\beta$-lactoglobulin with increased affinity for tri-cyclic drugs (e.g. antipsychotics and antidepressants) may be used in the future as molecular transporters that selectively recognize and remove toxic compounds from the body.

New BLG mutants with substitutions at positions 39, 56, 58, 71, 92, 105, and 107 were expressed in E.coli. All proteins were purified by anion exchange and size-exclusion chromatography. Crystals were obtained by the vapor diffusion method in the hanging drop setup. New BLG mutants, possessing the different shape of the binding pocket, were co-crystalized with tricyclic drugs (e.g. fluphenazine, clomipramine, and chlorpromazine) and selected fatty acids. X-ray diffraction data were collected at XtaLAB Synergy

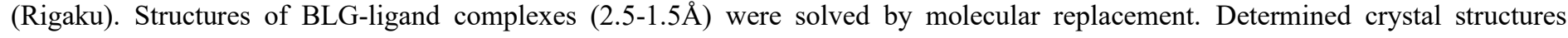
revealed that some substitutions reduced the length of the binding pocket preventing fatty acids from binding at this site. Crystallographic studies were supported by biophysical studies (circular dichroism) which allowed to determine the binding constant for selected protein-ligand complexes.

[1] Sawyer, L., Kontopidis, G. (2000). Acta - Protein Struct. Mol. Enzymol. 1482, 136-148.

[2] Pérez, M.D., Calvo, M. (1995). J. Dairy Sci. 78, 978-88.

[3] Sawyer, L. (2013). Advanced Dairy Chemistry, edited by P. L. H. McSweeney \& P. F. Fox, pp. 211-259, US Springer

[4] Rothe, C., Skerra, A. (2018). BioDrugs 32, 233-243.

[5] Deuschle, F.C., Ilyukhina, E., Skerra, A. (2020). Expert Opin Biol Ther. 21, 509-518.

Keywords: lactoglobulin, mutation, tricyclic drugs, crystal structure 